TRANSACTIONS OF THE

AMERICAN MATHEMATICAL SOCIETY

Volume 353, Number 10, Pages 4123-4138

S 0002-9947(01)02758-1

Article electronically published on June 6, 2001

\title{
CONDITIONAL STABILITY ESTIMATION FOR AN INVERSE BOUNDARY PROBLEM WITH NON-SMOOTH BOUNDARY IN $\mathcal{R}^{3}$
}

\author{
J. CHENG, Y. C. HON, AND M. YAMAMOTO
}

\begin{abstract}
In this paper, we investigate an inverse problem of determining a shape of a part of the boundary of a bounded domain in $\mathcal{R}^{3}$ by a solution to a Cauchy problem of the Laplace equation. Assuming that the unknown part is a Lipschitz continuous surface, we give a logarithmic conditional stability estimate in determining the part of boundary under reasonably a priori information of an unknown part. The keys are the complex extension and estimates for a harmonic measure.
\end{abstract}

\section{INTRODUCTION}

Assume that an inaccessible part of the boundary of an object in the three dimensional space was damaged or transformed with, for example, corrosion or continuous steel casting. A non-destructive evaluation technique is used for determining the shape of the corrosive or transformed boundary by a suitable observation on other accessible parts of the boundary. This is one inverse problem and is currently available in the engineering industry, and its solution is very important and in high demand both academically and practically. In this paper, the inverse problem of determining an unknown part of the boundary from overspecified boundary data is investigated.

The inverse problem can be formulated as follows: Assume that $\Omega \subset \mathcal{R}^{3}$ is a bounded domain. Let $\gamma \subset \partial \Omega$ be an unknown subboundary to be determined and $\Gamma$ be a known subboundary of $\partial \Omega$ where some observations can be made. It is not necessary that $\gamma \cup \Gamma=\partial \Omega$.

Consider a static field in $\Omega$ with a suitable function $u=u(x, y, z),(x, y, z) \in$ $\Omega \subset \mathcal{R}^{3}$. Throughout this paper, we assume that $u$ takes a given constant value on $\gamma$. This condition is reasonable, in the study of a convectively cooling continuously casting problem where the temperature $u$ must be the melting point on $\gamma$ (e.g. Siegel [26]). Without loss of generality, we may assume that the melting point is

Received by the editors July 27, 1999 and, in revised form, June 16, 2000.

1991 Mathematics Subject Classification. Primary 35R30, 31B20.

Key words and phrases. Determination of unknown boundary, conditional stability estimation, non-smooth boundary.

The first author is partly supported by NSF of China (No.19971016). This work was also partially supported by the Research Grants Council of the Hong Kong SAR, China (Grant numbers \#9040428) and the Sanwa Systems Development Company Limited (Tokyo, Japan).

(C)2001 American Mathematical Society 
zero, that is, $\left.u\right|_{\gamma}$ is zero. Thus the governing equations are given as

$$
\begin{aligned}
\Delta u(x, y, z) & =0, \quad(x, y, z) \in \Omega, \\
u(x, y, z) & =0, \quad(x, y, z) \in \gamma, \\
u(x, y, z) & =f(x, y, z), \quad(x, y, z) \in \Gamma,
\end{aligned}
$$

where $f$ is an input function which does not vanish identically on $\Gamma$.

The inverse problem of determining the transformed subboundary $\gamma$ is then stated as: Does

$$
\frac{\partial u}{\partial \nu}(x, y, z), \quad(x, y, z) \in \Gamma
$$

\section{determine $\gamma$ ?}

Here $\nu$ is the outward normal to the boundary $\Gamma$ and $\frac{\partial}{\partial \nu}$ denotes the normal derivative.

Similar problems in determining surface-breaking cracks were discussed by McIver [18 and Micheal, Waechter \& Collins [19. Related results can be found in Andrieux, Abda \& Jaoua [2], Aparicio \& Pidcock [3] and Kaup, Santosa \& Vogelius 14. In all of these papers, the results were only given for the two dimensional case. This paper investigates the inverse problem in the three dimensional case.

Since the governing equation (1.1) is the Laplace equation, the uniqueness of this inverse problem can be easily obtained by the unique continuation property for the Laplace equation (e.g. Mizohata [20]).

The difficulty in investigating this inverse problem is its severe ill-posedness. Since the boundary data are given on only a fixed part of the boundary, the illposedness of a Cauchy problem for the Laplace equation ([17], [27]) induces the ill-posedness of this inverse problem. Therefore a weaker conditional stability estimation for this problem is obtained by imposing some geometric restrictions on the shape of the transformed boundary. Such restrictions are reasonable from the practical viewpoint and can be imposed by assuming a boundedness condition on the geometric quantities of a subboundary. In general, rates of the conditional stability may depend on our choice of the geometric restrictions. To the authors' knowledge, even in the two dimensional case, there are not many similar conditional stability results for this kind of inverse problem. Futher references can be referred from the papers of Beretta and Vessela [4, Bukhgeim, Cheng and Yamamoto [5], 6], [7, Rondi [24, 25]. In our recent paper [5, a similar inverse problem in $\mathcal{R}^{2}$ was investigated and various conditional stability estimates were obtained within the $C^{2}$-regularity assumptions on the unknown subboundaries. This paper discusses conditional stability in the case of $\mathcal{R}^{3}$ where the unknown subboundaries are given by Lipschitz continuous functions with uniformly bounded Lipschitz constants. These geometric restrictions allow us to treat non-smooth surfaces with conical points, for example, as $\gamma$.

In relation to stability estimates in the determination of geometry, we refer to Alessandrini [1] for a crack determination problem, and Isakov [12], 13] and Ramm [23] for an inverse obstacle scattering problem. In Alessandrini [1, a logarithmic stability estimate was given when the boundary input is a combination of Dirac delta functions. The results given in this paper may be applicable to the crack determination problem.

The main result in this paper is a logarithmic stability under a priori assumptions. Although the rate is very weak, it holds generally for the determination of 
non-smooth subboundaries. Since real transformation processes such as corrosion usually yield non-smooth interfaces, our result is more practical in solving real industrial problems. Moreover, the paper for the crack problem [1] suggests that also in our inverse problems, the logarithmic rate cannot be improved in general, but may be improved for some special data.

This paper is organized as follows. The main theorem on stability estimation is given in Section 2. In Section 3, the main theorem is reduced to two key lemmata. The proofs of these key lemmata are then given in Sections 4 and 5 respectively.

\section{Main Result}

Let $\Omega_{1}, \Omega_{2}$ be ordinate sets whose upper subboundaries are assumed to be unknown after corrosion for example. Let $\Sigma_{1}, \Sigma_{2}$ be simply connected domains in $\mathcal{R}^{2}$ such that $\Sigma_{1} \subset \Sigma_{2}$ and $\operatorname{dist}\left(\partial \Sigma_{1}, \partial \Sigma_{2}\right)>0$.

For an arbitrarily fixed smooth function $\lambda=\lambda(x, y)>0$ defined on $\bar{\Sigma}_{2} \backslash \Sigma_{1}$ and positive constants $m_{0}, m_{1}$, we consider

$$
\begin{aligned}
& \mathcal{F}=\mathcal{F}\left(\lambda, m_{0}, m_{1}\right) \\
& =\left\{F \in C\left(\bar{\Sigma}_{2}\right)|\quad F|_{\Sigma_{2} \backslash \Sigma_{1}}=\lambda(x, y), \quad\left|F\left(x_{1}, y_{1}\right)-F\left(x_{2}, y_{2}\right)\right|\right. \\
& \left.\quad \leq m_{1}\left[\left|x_{1}-y_{1}\right|+\left|x_{2}-y_{2}\right|\right], \text { for }\left(x_{1}, y_{1}\right),\left(x_{2}, y_{2}\right) \in \bar{\Sigma}_{2} ; F>m_{0}, \text { on } \bar{\Sigma}_{2}\right\} .
\end{aligned}
$$

For $F_{1}, F_{2} \in \mathcal{F}$, we set

$$
\begin{aligned}
& \Omega_{1}=\left\{(x, y, z) ;(x, y) \in \Sigma_{2}, \quad 0<z<F_{1}(x, y)\right\} \\
& \Omega_{2}=\left\{(x, y, z) ;(x, y) \in \Sigma_{2}, \quad 0<z<F_{2}(x, y)\right\} .
\end{aligned}
$$

In other words, $\gamma_{j}=\left\{(x, y, z) ; z=F_{j}(x, y), \quad(x, y) \in \Sigma_{1}\right\}, j=1,2$, are unknown upper subboundaries to be determined and are given by Lipschitz continuous functions. The a priori information $F_{1}, F_{2} \in \mathcal{F}$ mean that the defect process did not make the shape of subboundaries extremely complicated.

The observation boundary is defined by

$$
\Gamma=\left\{(x, y, 0) ;(x, y) \in \Sigma_{3}\right\}
$$

where $\Sigma_{3} \subset \Sigma_{2}$ is a domain in $\mathcal{R}^{2}$.

Assume that $u_{j}, j=1,2$, satisfy

$$
\begin{gathered}
\Delta u_{j}(x, y, z)=0, \quad(x, y, z) \in \Omega_{j}, \\
u_{j}(x, y, z)=0, \quad(x, y, z) \in \gamma_{j}, \\
u_{j}(x, y, 0)=f_{j}(x, y), \quad \frac{\partial u_{j}}{\partial y}(x, y, 0)=g_{j}(x, y), \quad(x, y) \in \Sigma_{3} .
\end{gathered}
$$

The main result for the stability estimation is then stated in the following.

Theorem 2.1. Let $m_{2}>0,0<\alpha<1$, and $M>0$ be arbitrarily fixed constants. Assume that there exists a fixed point $\left(x^{0}, y^{0}, 0\right)$ such that

$$
\left|f_{j}\left(x^{0}, y^{0}\right)\right|>m_{2}, \quad j=1,2,
$$

and

$$
u_{j} \in C^{2}\left(\Omega_{j}\right) \cap C^{1+\alpha}\left(\bar{\Omega}_{j}\right), \quad\left\|u_{j}\right\|_{C^{1+\alpha}\left(\bar{\Omega}_{j}\right)} \leq M, \quad j=1,2 .
$$


Then there exists constants $C>0$ and $0<\tau<1$ depending on $\alpha, m_{0}, m_{1}, m_{2}$ and $M$ such that

$$
\left\|F_{1}-F_{2}\right\|_{C\left(\bar{\Sigma}_{1}\right)} \leq C\left|\frac{1}{\log \left(\log \frac{1}{\epsilon}\right)}\right|^{\tau}
$$

where $\epsilon=\left\|f_{1}-f_{2}\right\|_{L^{2}\left(\Sigma_{3}\right)}+\left\|g_{1}-g_{2}\right\|_{L^{2}\left(\Sigma_{3}\right)}$.

This double "log" estimate shows very weak stability and is observed in similar determination problems (e.g. Alessandrini [1], Isakov [12]). We notice that our estimate (2.10) is the worst possible and does not exclude possibilities of improvement of stability rate for some data set.

Remark 2.1. The assumption (2.8) requires that the Cauchy data do not identically vanish on $\Gamma$. It is necessary for obtaining the conditional stability estimation.

Remark 2.2. In Theorem 2.1 since $\left\|u_{j}\right\|_{C^{1+\alpha}\left(\bar{\Omega}_{j}\right)} \leq M$, there exist some constants $C^{\prime}>0$ and $0<\alpha_{1} \leq 1$ which depend on $M, \alpha$ such that

$$
\begin{aligned}
& \frac{1}{C^{\prime}}\left(\left\|f_{1}-f_{2}\right\|_{H^{1}\left(\Sigma_{3}\right)}+\left\|g_{1}-g_{2}\right\|_{L^{2}\left(\Sigma_{3}\right)}\right)^{\alpha_{1}} \leq \epsilon \\
& \leq C^{\prime}\left(\left\|f_{1}-f_{2}\right\|_{H^{1}\left(\Sigma_{3}\right)}+\left\|g_{1}-g_{2}\right\|_{L^{2}\left(\Sigma_{3}\right)}\right) .
\end{aligned}
$$

\section{Proof of Theorem 2.1}

Let $\left|F_{2}(x, y)-F_{1}(x, y)\right|$ attain its maximum at $\left(x^{*}, y^{*}\right) \in \Sigma_{1}$. Without loss of generality, assume that

$$
F_{2}\left(x^{*}, y^{*}\right)-F_{1}\left(x^{*}, y^{*}\right)=\left\|F_{1}-F_{2}\right\|_{C\left(\bar{\Sigma}_{1}\right)} .
$$

Let $\mathcal{D}$ be the connected component of $\Omega_{2} \backslash \Omega_{1}$ which includes a segment of the line $x=x^{*}, \quad y=y^{*}$. Let

$$
\Gamma_{1}=\partial \mathcal{D} \cap \partial \Omega_{1}, \quad \Gamma_{2}=\partial \mathcal{D} \cap \partial \Omega_{2} .
$$

Notice that $\partial \mathcal{D}=\Gamma_{1} \cup \Gamma_{2}$. We will prove

Lemma 3.1. There exist constants $0<\tau_{1} \leq 1$ and $C_{1}>0$ depending on $\Sigma_{1}, \Sigma_{2}$, $\Sigma_{3}, m_{0}, m_{1}, m_{2}, M$ and $\alpha$ such that

$$
\left\|u_{2}\right\|_{C\left(\Gamma_{1}\right)} \leq \frac{C_{1}}{\left(\log \frac{1}{\epsilon}\right)^{\tau_{1}}} \equiv \delta .
$$

Lemma 3.2. There exist constants $0<\tau \leq 1$ and $C_{2}>0$ depending on $\Sigma_{1}, \Sigma_{2}$, $\Sigma_{3}, m_{0}, m_{1}, m_{2}, M$ and $\alpha$ such that

$$
\left\|F_{1}-F_{2}\right\|_{C\left(\bar{\Sigma}_{1}\right)} \leq C_{2}\left[\frac{1}{\log \frac{1}{\delta}}\right]^{\tau} .
$$

The proof of Theorem 2.1] is then a direct consequence of Lemmata 3.1 and 3.2 We note that it is sufficient to prove the theorem for sufficiently small $\epsilon>0$. Then

$$
\begin{aligned}
\left\|F_{1}-F_{2}\right\|_{C\left(\bar{\Sigma}_{1}\right)} & \leq C_{2}\left[\frac{1}{\log \left(\frac{\left(\log \frac{1}{\epsilon}\right)^{\tau}}{C_{1}}\right)}\right]^{\tau} \\
& \leq C_{2}\left[\frac{1}{\tau_{1} \log \left(\log \frac{1}{\epsilon}\right)-\log C_{1}}\right]^{\tau} \\
& \leq C\left[\frac{1}{\log \left(\log \frac{1}{\epsilon}\right)}\right]^{\tau} .
\end{aligned}
$$




\section{Proof of Lemma 3.1}

Lemma 3.1 asserts conditional stability of log-order rate. Such an estimate is common for the Cauchy problem of the Laplace equation (e.g. Lavrentiev [17, Payne [21]). For our purpose, we further have to prove the stability which holds uniformly in every domain whose upper boundary is given by $F \in \mathcal{F}$. First we prove

Lemma 4.1. There exist constants $C_{3}>0$ and $0<\chi<1$ which are independent of $z$ such that

$$
\left|u_{2}\left(x_{1}, y_{1}, z\right)-u_{1}\left(x_{1}, y_{1}, z\right)\right| \leq C_{3} \epsilon^{\chi}, \quad\left(x_{1}, y_{1}\right) \in \bar{\Sigma}_{1}, \quad z \in\left[\frac{m_{0}}{4}, \frac{m_{0}}{2}\right] .
$$

In fact, $\inf \left\{\operatorname{dist}\left(\left(x_{1}, y_{1}, z\right) ; \partial \Omega_{1}\right) ; \frac{m_{0}}{4} \leq z \leq \frac{m_{0}}{2}\right\}>\frac{m_{0}}{8}>0$ and so an interior estimate for the Cauchy problem of the Laplace equations yields (4.1) (e.g. Lavrentiev [17, Payne [21]).

Let

$$
E=\left\{\zeta=\xi_{1}+i \xi_{2} \in \mathcal{C}|\quad| \zeta|<R,| \arg \zeta \mid<\theta\right\}
$$

and $l=\left[\rho_{1}, \rho_{2}\right] \subset E$.

Definition 4.1. $\psi(\zeta)$ is called the harmonic measure for $E$ and $l$ if it satisfies

$$
\begin{aligned}
\Delta \psi(\zeta) & =0, & & \zeta \in E \backslash l, \\
\psi(\zeta) & =0, & & \zeta \in \partial E, \\
\psi(\zeta) & =1, & & \zeta \in l .
\end{aligned}
$$

We refer to Friedman and Vogelius [10, Kellog [15] for the existence and uniqueness of the harmonic measure. Moreover by [10], we have $\psi \in C(\bar{E})$.

Lemma 4.2. The harmonic measure $\psi$ for $E$ and $l$ satisfies the following estimation:

$$
\psi\left(\xi_{1}\right) \geq C_{4}\left(\left(\frac{\rho_{2}}{\xi_{1}}\right)^{\frac{\pi}{2 \theta}}-\left(\frac{\rho_{2}}{R}\right)^{\frac{\pi}{2 \theta}}\right), \quad \xi_{1} \in\left[\rho_{2}, R\right],
$$

where $C_{4}=C_{4}\left(R, \theta, \rho_{1}, \rho_{2}\right)>0$ is a constant which is independent of $\xi_{1}$.

Moreover, for an arbitrarily fixed $\mu>1$, we can take a constant $C_{4}(\mu)>0$ which is dependent on $\mu$ but independent of $R, \theta, \rho_{1}, \rho_{2}$, provided that

$$
\left(\frac{\rho_{2}}{\rho_{1}}\right)^{\frac{\pi}{2 \theta}}-\left(\frac{\rho_{2}}{R}\right)^{\frac{\pi}{2 \theta}} \geq \mu
$$

Proof. Henceforth $\Re \zeta$ and $\Im \zeta$ denote the real part and the imaginary part of $\zeta \in \mathcal{C}$ respectively. We can prove the lemma in a way similar to Isakov [12]. That is, consider the conformal map

$$
\eta=\phi(\zeta)=\left(\frac{\rho_{2}}{\zeta}\right)^{\frac{\pi}{2 \theta}}
$$

which transforms the domain $E \backslash l$ to

$$
S=\left\{\eta|\Re \eta>0,| \eta \mid>\left(\frac{\rho_{2}}{R}\right)^{\frac{\pi}{2 \theta}}\right\} \backslash\left\{\eta=t \mid t \in\left[1,\left(\frac{\rho_{2}}{\rho_{1}}\right)^{\frac{\pi}{2 \theta}}\right]\right\} .
$$

Therefore the function $w(\eta)=\psi\left(\phi^{-1}(\eta)\right)$ satisfies

$$
\begin{aligned}
\Delta w(\eta) & =0, & & \eta \in S, \\
w(\eta) & =0, & & \eta \in \phi(\partial E), \\
w(\eta) & =1, & & \eta \in\left[1,\left(\frac{\rho_{2}}{\rho_{1}}\right)^{\frac{\pi}{2 \theta}}\right] .
\end{aligned}
$$


We note that

$$
\phi(\partial E)=\left\{\eta|\Re \eta>0,| \eta \mid=\left(\frac{\rho_{2}}{R}\right)^{\frac{\pi}{2 \theta}}\right\} \cup\left\{\eta|\Re \eta=0,| \eta \mid>\left(\frac{\rho_{2}}{R}\right)^{\frac{\pi}{2 \theta}}\right\} .
$$

By the maximum principle, we see

$$
0<w<1, \quad \text { in } S \text {. }
$$

Let $l_{1}=\left[1-\left(\frac{\rho_{2}}{R}\right)^{\frac{\pi}{2 \theta}},\left(\frac{\rho_{2}}{\rho_{1}}\right)^{\frac{\pi}{2 \theta}}-\left(\frac{\rho_{2}}{R}\right)^{\frac{\pi}{2 \theta}}\right]$ and let $w_{0}(\eta)$ satisfy

$$
\begin{aligned}
\Delta w_{0}(\eta) & =0, \quad\{\Re \eta>0\} \backslash l_{1}, \\
w_{0}(\eta) & =0, \quad \Re \eta=0, \\
w_{0}(\zeta) & =1, \quad \zeta \in l_{1}, \\
w_{0}(\infty) & =0 .
\end{aligned}
$$

By the maximum principle in [22], we have

$$
w(\eta) \geq w_{0}\left(\eta-\left(\frac{\rho_{2}}{R}\right)^{\frac{\pi}{2 \theta}}\right), \quad \Re \eta \geq\left(\frac{\rho_{2}}{R}\right)^{\frac{\pi}{2 \theta}},
$$

and

$$
0<w_{0}(\eta)<1, \quad\{\Re \eta>0\} \backslash l_{1} .
$$

By the strong maximum principle (e.g. [22]), we have

$$
\frac{\partial w_{0}}{\partial \xi_{1}}(0,0) \neq 0 \text {. }
$$

Therefore there exists a constant $C_{4}^{\prime}>0$ such that

$$
w_{0}(\eta) \geq C_{4}^{\prime} \eta, \quad \Im \eta=0, \quad 0 \leq \eta \leq 1 .
$$

In fact, assume to the contrary that (4.12) is not true. Then there exist $y_{n} \in$ $[0,1], n=1,2, \cdots$, such that $\frac{w_{0}\left(y_{n}\right)}{y_{n}} \rightarrow 0$ as $n \rightarrow \infty$. If necessary, we can take a subsequence, so that there exists $\widetilde{y} \in[0,1]$ such that $y_{n} \rightarrow \widetilde{y}$ as $n \rightarrow \infty$.

If $\widetilde{y}=0$, then $\frac{\partial w_{0}}{\partial \xi_{1}}(0,0)=0$, which contradicts (4.11).

If $\widetilde{y} \neq 0$, then the continuity of $w_{0}$ implies $w_{0}(\widetilde{y})=0$, which contradicts (4.10).

It is obvious that $C_{4}^{\prime}$ depends on $\rho_{1}, \rho_{2}$ and $R, \theta$. In terms of $\phi(\zeta)=\left(\frac{\rho_{2}}{\zeta}\right)^{\frac{\pi}{2 \theta}}$, the inequalities (4.9) and (4.12) mean (4.3).

If $\left(\frac{\rho_{2}}{\rho_{1}}\right)^{\frac{\pi}{2 \theta}}-\left(\frac{\rho_{2}}{R}\right)^{\frac{\pi}{2 \theta}} \geq \mu>1$, then $l_{1} \supset[1, \mu]$. Let $\widetilde{w}_{0}$ be the solution of (4.5) - (4.8) where $l_{1}$ is replaced by $[1, \mu]$. Then in terms of $l_{1} \supset[1, \mu]$, the maximum principle implies that $w_{0}(\eta) \geq \widetilde{w}_{0}(\eta), \Re \eta>0$. Since $\widetilde{w}_{0}$ is independent of $\rho_{1}, \rho_{2}$ and $R$, an estimate for $\widetilde{w}_{0}$ similar to (4.12) concludes that $C_{4}$ can be chosen independently of $\rho_{1}, \rho_{2}$ and $R$ as long as (4.4) is satisfied. The proof is complete.

Lemma 4.3. (i) The harmonic measure $\psi$ for $E$ and $l$ satisfies

$$
\psi\left(\xi_{1}\right) \geq C_{5} \xi^{\tau_{2}}, \quad \xi_{1} \in\left[0, \rho_{1}\right],
$$

where $C_{5}=C_{5}\left(R, \theta, \rho_{1}, \rho_{2}\right)>0$ is independent of $\xi_{1}$ and a constant $0<\tau_{2}<1$ depends only on $\theta$.

(ii) For any bounded interval $I \subset\left(\rho_{2}, \infty\right)$, we have

$$
\inf _{R \in I} C_{5}\left(R, \theta, \rho_{1}, \rho_{2}\right)>0 .
$$

In other words, we can take $C_{5}>0$ uniformly with respect to $R$ provided that $R$ varies in any bounded interval in $\left(\rho_{2}, \infty\right)$. 
Proof. (i) The conformal map

$$
\eta_{1}=\phi_{1}(\zeta)=\left(\frac{\zeta}{R}\right)^{\frac{\pi}{2 \theta}}
$$

transforms the domain $E \backslash l$ to

$$
\mathcal{S}_{1}(R)=\{\eta|| \eta \mid<1, \Re \eta>0\} \backslash\left[\left(\frac{\rho_{1}}{R}\right)^{\frac{\pi}{2 \theta}},\left(\frac{\rho_{2}}{R}\right)^{\frac{\pi}{2 \theta}}\right] .
$$

Therefore the function $w_{1}(\eta ; R)=\psi\left(\phi_{1}^{-1}(\eta)\right)$ satisfies

$$
\begin{aligned}
\Delta w_{1}(\eta ; R) & =0, \quad \eta \in \mathcal{S}_{1}(R), \\
w_{1}(\eta ; R) & =0, \quad \eta \in \phi_{1}(\partial E), \\
w_{1}(\eta ; R) & =1, \quad\left(\frac{\rho_{1}}{R}\right)^{\frac{\pi}{2 \theta}}<\eta<\left(\frac{\rho_{2}}{R}\right)^{\frac{\pi}{2 \theta}} .
\end{aligned}
$$

Similarly to the proof for Lemma 4.2 , we have

$$
w_{1}(\eta ; R) \geq C_{5} \eta, \quad \eta \in\left[0,\left(\frac{\rho_{1}}{R}\right)^{\frac{\pi}{2 \theta}}\right] .
$$

Return to $\psi$ and the proof of the part (i) is proved.

(ii) We set $I=\left[R_{0}, R_{1}\right]$ where $R_{0}>\rho_{2}$. Let us put $2 \nu=\frac{\rho_{2}}{\rho_{1}}-1>0$.

Let $\widetilde{w}^{1}(\eta)$ satisfy

$$
\begin{aligned}
\Delta \widetilde{w}^{1}(\eta) & =0, \quad \eta \in\{\eta|| \eta \mid<1, \Re \eta>0\} \backslash\left[\left(\frac{\rho_{1}}{R_{0}}\right)^{\frac{\pi}{2 \theta}},\left(\frac{\rho_{2}}{(1+\nu) R_{0}}\right)^{\frac{\pi}{2 \theta}}\right] \\
\widetilde{w}^{1}(\eta) & =0, \quad \eta \in \phi_{1}(\partial E) \\
\widetilde{w}^{1}(\eta) & =1, \quad\left(\frac{\rho_{1}}{R_{0}}\right)^{\frac{\pi}{2 \theta}}<\eta<\left(\frac{\rho_{2}}{(1+\nu) R_{0}}\right)^{\frac{\pi}{2 \theta}}
\end{aligned}
$$

We note that

$$
1+\nu<1+2 \nu=\frac{\rho_{2}}{\rho_{1}}
$$

implies

$$
\left(\frac{\rho_{1}}{R_{0}}\right)^{\frac{\pi}{2 \theta}}<\left(\frac{\rho_{2}}{(1+\nu) R_{0}}\right)^{\frac{\pi}{2 \theta}}
$$

so that $\widetilde{w}^{1}$ is well-defined.

For $R \in\left[R_{0},(1+\nu) R_{0}\right]$, we have

$$
\left(\frac{\rho_{1}}{R}\right)^{\frac{\pi}{2 \theta}} \leq\left(\frac{\rho_{1}}{R_{0}}\right)^{\frac{\pi}{2 \theta}}<\left(\frac{\rho_{2}}{(1+\nu) R_{0}}\right)^{\frac{\pi}{2 \theta}} \leq\left(\frac{\rho_{2}}{R}\right)^{\frac{\pi}{2 \theta}} .
$$

Therefore by the maximum principle, we obtain

$$
\inf _{R_{0} \leq R \leq(1+\nu) R_{0}} w_{1}(\eta ; R) \geq \widetilde{w}^{1}(\eta)
$$

for $\eta \in\left[0,\left(\frac{\rho_{1}}{R}\right)^{\frac{\pi}{2 \theta}}\right]$.

Similarly to the proof of Lemma 4.2, we have

$$
\widetilde{w}^{1}(\eta) \geq C_{5}^{(1)} \eta, \quad \eta \in\left[0,\left(\frac{\rho_{1}}{R}\right)^{\frac{\pi}{2 \theta}}\right],
$$

where $C_{5}^{(1)}>0$ is dependent on $\theta, \rho_{1}$ and $\rho_{2}$, but independent of $R \in\left[R_{0},(1+\nu) R_{0}\right]$.

Therefore we have

$$
\inf _{R_{0} \leq R \leq(1+\nu) R_{0}} w_{1}(\eta ; R) \geq C_{5}^{(1)} \eta, \quad \eta \in\left[0,\left(\frac{\rho_{1}}{R}\right)^{\frac{\pi}{2 \theta}}\right] .
$$


Next, for $R \in\left((1+\nu) R_{0},(1+\nu)^{2} R_{0}\right]$, we have

$$
\left(\frac{\rho_{1}}{R}\right)^{\frac{\pi}{2 \theta}} \leq\left(\frac{\rho_{1}}{(1+\nu) R_{0}}\right)^{\frac{\pi}{2 \theta}}<\left(\frac{\rho_{2}}{(1+\nu)^{2} R_{0}}\right)^{\frac{\pi}{2 \theta}} \leq\left(\frac{\rho_{2}}{R}\right)^{\frac{\pi}{2 \theta}},
$$

so that a similar argument implies that we can take a constant $C_{5}^{(2)}=C_{5}^{(2)}\left(\theta, \rho_{1}, \rho_{2}\right)$ $>0$ independent of $R \in\left[(1+\nu) R_{0},(1+\nu)^{2} R_{0}\right]$ such that

$$
\inf _{(1+\nu) R_{0} \leq R \leq(1+\nu)^{2} R_{0}} w_{1}(\eta ; R) \geq C_{5}^{(2)} \eta, \quad \eta \in\left[0,\left(\frac{\rho_{1}}{R}\right)^{\frac{\pi}{2 \theta}}\right] .
$$

Continuing this argument until $(1+\nu)^{m} R_{0} \geq R_{1}$ with some natural number $m$, we see

$$
\inf _{R_{0} \leq R \leq R_{1}} w_{1}(\eta ; R) \geq \min _{1 \leq j \leq m} C_{5}^{(j)} \eta, \quad \eta \in\left[0,\left(\frac{\rho_{1}}{R}\right)^{\frac{\pi}{2 \theta}}\right] .
$$

Return to $\psi$ and we can complete the proof of the lemma.

By Lemmata 4.2 and 4.3 , we show the following conditional stability estimation for a holomorphic function in $E$.

Lemma 4.4. Suppose that $v=v(\zeta)$ is a holomorphic function in $E$. Let $\varepsilon=$ $\max _{\xi_{1} \in\left[\rho_{1}, \rho_{2}\right]}\left|v\left(\xi_{1}\right)\right|$. If $|v(\zeta)| \leq M_{1}$ for $\zeta \in E$, then

$$
\left|v\left(\xi_{1}\right)\right| \leq M_{1}\left(\frac{\varepsilon}{M_{1}}\right)^{C_{4}\left(\left(\frac{\rho_{2}}{\xi_{1}}\right)^{\frac{\pi}{2 \theta}}-\left(\frac{\rho_{2}}{R}\right)^{\frac{\pi}{2 \theta}}\right)} \leq M_{1} \varepsilon^{C_{4}\left(\left(\frac{\rho_{2}}{\xi_{1}}\right)^{\frac{\pi}{2 \theta}}-\left(\frac{\rho_{2}}{R}\right)^{\frac{\pi}{2 \theta}}\right)}, \quad \xi_{1} \in\left[\rho_{2}, R\right],
$$

and

$$
\left|v\left(\xi_{1}\right)\right| \leq M_{1}\left(\frac{\varepsilon}{M_{1}}\right)^{C_{5} \xi^{\tau_{2}}} \leq M_{1} \varepsilon^{C_{5} \xi^{\tau_{2}}}, \quad \xi_{1} \in\left[0, \rho_{1}\right] .
$$

Proof. Consider the function

$$
M(\zeta)=|v(\zeta)| e^{\psi(\zeta) \log M_{1}+(1-\psi(\zeta)) \log \varepsilon} .
$$

We will prove that

$$
M(\zeta) \leq \max _{z \in \partial E \cup\left[\rho_{1}, \rho_{2}\right]} M(z)=M_{1} \varepsilon \quad \text { for } \quad \text { any } \quad \zeta \in \bar{E} .
$$

In fact, the second equality of (4.24) is readily seen by noting that $\max _{\zeta \in \partial E}|v(\zeta)|=$ $M_{1}$ and the maximum principle of a holomorphic function. Assume that the first inequality of (4.24) is not true. Then there exists $\zeta_{0} \in E \backslash\left[\rho_{1}, \rho_{2}\right]$ such that

$$
M\left(\zeta_{0}\right)=\max _{\zeta \in \bar{E}} M(\zeta)>\max _{z \in \partial E \cup\left[\rho_{1}, \rho_{2}\right]} M(z)=M_{1} \varepsilon
$$

Let $\omega$ be the simply connected component of $E \backslash\{\Re \zeta=0\}$ which contains $\zeta_{0}$.

From the theory of complex variables, for the harmonic function $\psi(\zeta)$ in $\omega$, there exists a holomorphic function $\Psi(\zeta)$ in $\omega$ such that

$$
\Re \Psi(\zeta)=\psi(\zeta), \quad \zeta \in \omega,
$$

where $\Re \Psi(\zeta)$ is the real part of $\Psi(\zeta)$.

We set

$$
V(\zeta)=v(\zeta) e^{\Psi(\zeta) \log M_{1}+[1-\Psi(\zeta)] \log \varepsilon}, \quad \zeta \in \omega .
$$

Then $|V(\zeta)|=M(\zeta)$ for $\zeta \in \omega$. It is easy to verify that $V(\zeta)$ is a holomorphic function in $\omega$ and $V(\zeta)$ attains its maximum $M\left(\zeta_{0}\right)$ at an interior point $\zeta_{0}$. Therefore the maximum principle for a holomorphic function implies

$$
|V(\zeta)|=\text { constant }, \quad \zeta \in \omega .
$$




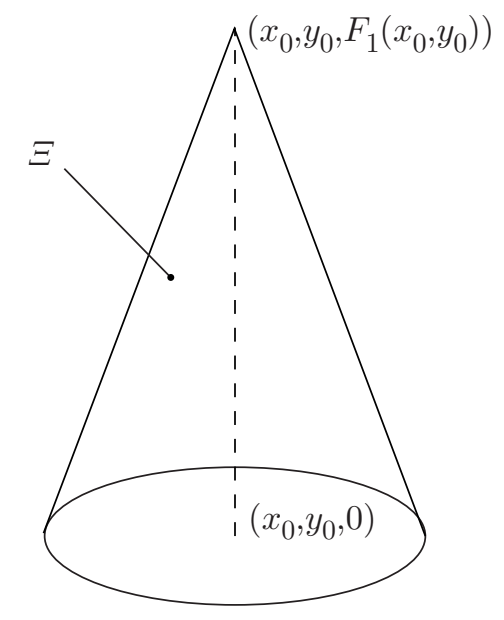

Figure 1. A cone with a vertex on $\Gamma_{1}$

Consequently

$$
M(\zeta)=\text { constant }, \quad \zeta \in \omega .
$$

By $\psi \in C(\bar{E})$, we see that

$$
M(\zeta)=\text { constant, } \quad \zeta \in \omega \cup\left[\rho_{1}, \rho_{2}\right] .
$$

This contradicts (4.25) and hence (4.24) must hold.

Hence by (4.24), we obtain

$$
\left|v\left(\xi_{1}\right)\right| \leq M_{1}^{1-\psi\left(\xi_{1}\right)} \varepsilon^{\psi\left(\xi_{1}\right)}, \quad \xi_{1} \in[0, R] .
$$

Noting $\left(\frac{\varepsilon}{M_{1}}\right) \leq 1$, we apply Lemmata 4.2 and 4.3 and complete the proof of the lemma.

Now we can complete the proof of Lemma 3.1.

For any $\left(x_{0}, y_{0}, F_{1}\left(x_{0}, y_{0}\right)\right) \in \Gamma_{1}$, there exists a constant $\beta>0$ which depends only on $m_{1}, \Sigma_{1}$ and $\Sigma_{2}$ such that

$$
\begin{aligned}
\Xi=\left\{(x, y, z) \in \mathcal{R}^{3} \mid\left\{\left|x-x_{0}\right|^{2}+\left|y-y_{0}\right|^{2}\right\}^{\frac{1}{2}}\right. & <\beta\left(F_{1}\left(x_{0}, y_{0}\right)-z\right), \\
& \left.0<z<F_{1}\left(x_{0}, y_{0}\right)\right\} \subset \Omega_{2}
\end{aligned}
$$

for any $\left(x_{0}, y_{0}, F_{1}\left(x_{0}, y_{0}\right)\right) \in \Gamma_{1}$ (see Figure 1$)$.

Let us set $u=u_{2}-u_{1}$ and

$$
\begin{aligned}
(4.27) v(\zeta) & =\int_{\partial \Xi}\left[G\left(\xi_{1}, \xi_{2}, \xi_{3}, x_{0}, y_{0}, \zeta\right) \frac{\partial u}{\partial n}\left(\xi_{1}, \xi_{2}, \xi_{3}\right)\right. \\
& \left.-\frac{\partial G}{\partial n}\left(\xi_{1}, \xi_{2}, \xi_{3}, x_{0}, y_{0}, \zeta\right) u\left(\xi_{1}, \xi_{2}, \xi_{3}\right)\right] d S, \quad 0<\zeta<F_{1}\left(x_{0}, y_{0}\right),
\end{aligned}
$$

where $d S$ is the area element with respect to $\left(\xi_{1}, \xi_{2}, \xi_{3}\right), n$ is the unit outer normal vector to $\partial \Xi$ and

$$
G\left(\xi_{1}, \xi_{2}, \xi_{3}, x_{0}, y_{0}, \zeta\right)=\frac{1}{4 \pi} \frac{1}{\left[\left(\xi_{1}-x_{0}\right)^{2}+\left(\xi_{2}-y_{0}\right)^{2}+\left(\xi_{3}-\zeta\right)^{2}\right]^{\frac{1}{2}}}
$$


Next we will verify that $v(\zeta)$ can be analytically extended and is holomorphic in

$$
\begin{aligned}
\Xi_{1} \equiv & \left\{\zeta \in \mathcal{C}\left|\quad 0<\Re \zeta<F_{1}\left(x_{0}, y_{0}\right), \quad\right| \zeta-F_{1}\left(x_{0}, y_{0}\right) \mid<F_{1}\left(x_{0}, y_{0}\right),\right. \\
& \left.\left|\arg \left(F_{1}\left(x_{0}, y_{0}\right)-\zeta\right)\right|<\arctan \beta\right\},
\end{aligned}
$$

and

$$
u\left(x_{0}, y_{0}, z\right)=v(z), \quad z \in\left[0, F\left(x_{0}, y_{0}\right)\right] .
$$

The equality (4.28) is straightforward from (4.27) and Green's representation formula (e.g. Chapter 2, $\S 2.4$ in Gilbarg and Trudinger [1]). Next we need to verify that $v$ is holomorphic in $\Xi_{1}$. We first examine the set $V$ :

$$
V=\left\{\zeta \in \mathcal{C} \mid\left(\xi_{1}-x_{0}\right)^{2}+\left(\xi_{2}-y_{0}\right)^{2}+\left(\xi_{3}-\zeta\right)^{2}=0, \quad\left(\xi_{1}, \xi_{2}, \xi_{3}\right) \in \partial \Xi\right\} .
$$

We rewrite $\left(\xi_{1}-x_{0}\right)^{2}+\left(\xi_{2}-y_{0}\right)^{2}+\left(\xi_{3}-\zeta\right)^{2}$ as $\left(\zeta-\xi_{3}+i r\right)\left(\zeta-\xi_{3}-i r\right)$ where $r=r\left(\xi_{1}, \xi_{2}\right)=\sqrt{\left(\xi_{1}-x_{0}\right)^{2}+\left(\xi_{2}-y_{0}\right)^{2}}$. Then we can see that

$$
\begin{aligned}
V & =\left\{\zeta \mid \quad \Re \zeta=\xi_{3}, \Im \zeta=r, \quad\left(\xi_{1}, \xi_{2}, \xi_{3}\right) \in \partial \Xi\right\} \\
& \cup \quad\left\{\zeta \mid \quad \Re \zeta=\xi_{3}, \Im \zeta=-r, \quad\left(\xi_{1}, \xi_{2}, \xi_{3}\right) \in \partial \Xi\right\} .
\end{aligned}
$$

Noting that

$$
\begin{aligned}
\partial \Xi & =\left\{\left(\xi_{1}, \xi_{2}, 0\right) \mid \quad r \leq \beta F_{1}\left(x_{0}, y_{0}\right)\right\} \\
& \cup\left\{\left(\xi_{1}, \xi_{2}, \xi_{3}\right) \mid r=\beta\left(F_{1}\left(x_{0}, y_{0}\right)-\xi_{3}\right), 0<\xi_{3} \leq F_{1}\left(x_{0}, y_{0}\right)\right\},
\end{aligned}
$$

we have that

$$
\begin{aligned}
V & =\left\{\zeta \mid \quad \Re \zeta=0, \quad-\beta F_{1}\left(x_{0}, y_{0}\right) \leq \Im \zeta \leq \beta F_{1}\left(x_{0}, y_{0}\right)\right\} \\
& \cup\left\{\zeta \mid \quad \Im \zeta=\beta\left(\Re \zeta-F_{1}\left(x_{0}, y_{0}\right)\right), \quad 0<\Re \zeta \leq F_{1}\left(x_{0}, y_{0}\right)\right\} \\
& \cup \quad\left\{\zeta \mid \quad \Im \zeta=-\beta\left(\Re \zeta-F_{1}\left(x_{0}, y_{0}\right)\right), \quad 0<\Re \zeta \leq F_{1}\left(x_{0}, y_{0}\right)\right\} .
\end{aligned}
$$

Consequently $V \cap \Xi_{1}=\emptyset$. Therefore if $\zeta \in \Xi_{1}$, then $\left(\xi_{1}-x_{0}\right)^{2}+\left(\xi_{2}-y_{0}\right)^{2}+\left(\xi_{3}-\zeta\right)^{2}$ does not vanish for any $\left(\xi_{1}, \xi_{2}, \xi_{3}\right) \in \partial \Xi$. Thus we have verified that $v$ is holomorphic in $\Xi_{1}$.

Let $0<h<\frac{m_{0}}{4}$ be a fixed constant. Estimating $v$ by (4.27) and (2.9), we have

$$
|v(\zeta)| \leq \frac{C_{6}(M)}{h^{2}}, \quad \zeta \in \Xi_{h},
$$

where $\Xi_{h}=\left\{\zeta \mid \zeta \in \Xi_{1}, \operatorname{dist}\left(\zeta, \partial \Xi_{1}\right) \geq h\right\}$ and the constant $C_{6}(M)>0$ depends on $M$.

By Lemma 4.1, we have that

$$
|v(\zeta)| \leq C_{3} \epsilon^{\chi}, \quad \text { for } \quad \Im \zeta=0, \quad \frac{m_{0}}{4} \leq \Re \zeta \leq \frac{m_{0}}{2} .
$$

Applying (4.23) in Lemma 4.4 to $\Xi_{h}$, we have

$$
|v(z)| \leq \frac{C_{6}(M)}{h^{2}} \epsilon^{C_{7}\left(F_{1}\left(x_{0}, y_{0}\right)-h-z\right)^{\tau_{2}}}
$$

for $\frac{m_{0}}{2} \leq z \leq F_{1}\left(x_{0}, y_{0}\right)-h$.

Moreover, we can take a constant $M_{0}\left(m_{1}, \lambda\right)$ which depends on $\lambda$ and $m_{1}$ in (2.1) and satisfies

$$
\frac{m_{0}}{2}<m_{0} \leq F_{1}\left(x_{0}, y_{0}\right) \leq M_{0}\left(m_{1}, \lambda\right) .
$$

Therefore by Lemma 4.3 (ii), we can choose a constant $C_{7}>0$ which is independent of $F_{1} \in \mathcal{F}$ and $\left(x_{0}, y_{0}\right)$. 
Now we will complete the estimation of $u_{2}\left(x_{0}, y_{0}, F_{1}\left(x_{0}, y_{0}\right)\right)$. In terms of

$$
u_{1}\left(x_{0}, y_{0}, F_{1}\left(x_{0}, y_{0}\right)\right)=0 \text {, }
$$

(2.9), (4.28) and (4.31), we obtain

$$
\begin{aligned}
\left|u_{2}\left(x_{0}, y_{0}, F_{1}\left(x_{0}, y_{0}\right)\right)\right| & =\left|u\left(x_{0}, y_{0}, F_{1}\left(x_{0}, y_{0}\right)\right)\right| \\
& \leq\left|u\left(x_{0}, y_{0}, F_{1}\left(x_{0}, y_{0}\right)\right)-u\left(x_{0}, y_{0}, z\right)\right|+\left|u\left(x_{0}, y_{0}, z\right)\right| \\
& \leq M\left|z-F\left(x_{0}, y_{0}\right)\right|+|v(z)| \\
& \leq M\left|z-F\left(x_{0}, y_{0}\right)\right|+\frac{C_{6}(M)}{h^{2}} \epsilon^{C_{7}\left(F_{1}\left(x_{0}, y_{0}\right)-h-z\right)^{\tau_{2}} .}
\end{aligned}
$$

Let us choose $z=F_{1}\left(x_{0}, y_{0}\right)-2 h$. Then we have

$$
\left|u_{2}\left(x_{0}, y_{0}, F_{1}\left(x_{0}, y_{0}\right)\right)\right| \leq 2 h M+\frac{C_{6}(M)}{h^{2}} \epsilon^{C_{7}} h^{\tau_{2}} .
$$

We take $h$ which minimizes $2 h M+\frac{C_{6}(M)}{h^{2}} \epsilon^{C_{7}} h^{\tau_{2}}$. Then we can obtain the conclusion of Lemma 3.1. The proof is complete.

\section{Proof of Lemma 3.2}

First, by the maximum principle, we have

$$
\left|u_{2}(x, y, z)\right| \leq \delta, \quad(x, y, z) \in \mathcal{D} .
$$

Let

$$
d=F_{2}\left(x^{*}, y^{*}\right)-F_{1}\left(x^{*}, y^{*}\right)=\left\|F_{2}-F_{1}\right\|_{C\left(\bar{\Sigma}_{1}\right)}, \quad \theta=\arctan \frac{m_{1}}{2} .
$$

We can prove that for every $F \in \mathcal{F}$, both the domains $\{(x, y, z) \mid z<F(x, y)\}$ and $\{(x, y, z) \mid z>F(x, y)\}$ contain a cone with vertex $\left(x^{*}, y^{*}, F_{1}\left(x^{*}, y^{*}\right)\right)$ and angle $2 \theta$ whose axes are perpendicular to the $x y$-plane, wherever $x^{*}, y^{*}$ are. That is,

$$
\sqrt{\left(x-x^{*}\right)^{2}+\left(y-y^{*}\right)^{2}}<\tan \theta\left(F\left(x^{*}, y^{*}\right)-z\right)
$$

implies $z<F(x, y)$ and

$$
\sqrt{\left(x-x^{*}\right)^{2}+\left(y-y^{*}\right)^{2}}<\tan \theta\left(z-F\left(x^{*}, y^{*}\right)\right)
$$

implies $z>F(x, y)$.

From the assumptions (2.8), there exists a positive constant $\sigma<m_{0}$ such that

$$
\left|u_{2}\left(x^{0}, y^{0}, \sigma\right)\right| \geq \frac{m_{2}}{2},
$$

where $\sigma$ is independent of $u_{2}$ and is dependent only on $M$ and $m_{2}$.

Without loss of generality, we can assume that $y^{0}=y^{*}=0$. Otherwise we can take the line connecting $y^{0}$ and $y^{*}$ as a new $y$-axis and the line perpendicular to the new $y$-axis as an $x$-axis.

Next we will show that there exist constants $\kappa>0, C_{8}>0$ and $C_{9}>0$ which depend only on $m_{0}, m_{1}, m_{2}$ and $M$ such that

$$
\left|u_{2}(x, 0, \sigma)\right| \leq C_{8} \delta^{C_{9} d^{\frac{\pi}{\theta}}} \equiv \delta_{1}, \quad x \in\left[-\kappa+x^{*}, \kappa+x^{*}\right] .
$$

Proof of (5.3). We set

$$
z^{*}=\frac{F_{1}\left(x^{*}, 0\right)+F_{2}\left(x^{*}, 0\right)}{2} .
$$




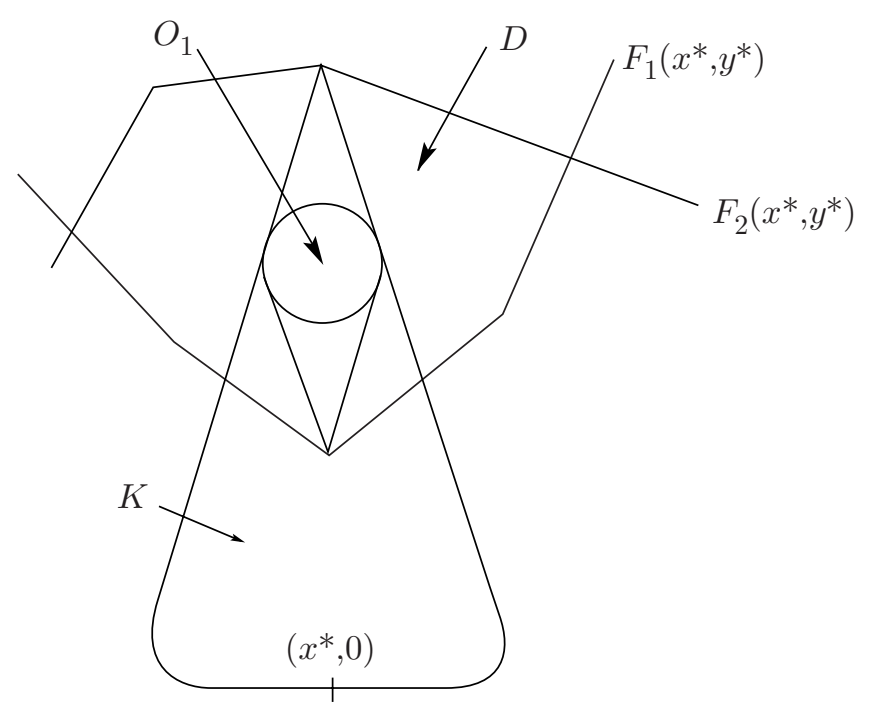

Figure 2. The cross section with $y=0$

Since $F_{j} \in \mathcal{F}$, there exists a small ball $O_{1}=O_{d_{1}}\left(x^{*}, 0, z^{*}\right)$ which centers at $\left(x^{*}, 0, z^{*}\right)$ with radius $d_{1}$ and satisfies $O_{d_{1}}\left(x^{*}, 0, z^{*}\right) \subset \mathcal{D}$ (see Figure 2$)$. Here

$$
d_{1}=\alpha_{2} d, \quad 0<\alpha_{2}=\frac{1}{2} \sin \theta<\frac{1}{2},
$$

and $\alpha_{2}$ depends on $m_{1}$.

Therefore from (5.1), we have

$$
\left|u_{2}(x, y, z)\right| \leq \delta, \quad(x, y, z) \in O_{1} .
$$

Let

$$
\begin{aligned}
\Xi_{2}= & \left\{(x, y, z) \mid\left[\left(x-x^{*}\right)^{2}+y^{2}\right]^{\frac{1}{2}}<\tan \theta\left(F_{2}\left(x^{*}, 0\right)-z\right),\right. \\
& \left.\sqrt{\left(x-x^{*}\right)^{2}+y^{2}+\left(z-F_{2}\left(x^{*}, 0\right)\right)^{2}}<F\left(x^{*}, 0\right)\right\}
\end{aligned}
$$

and let $B_{1}$ be one of connected components of $\Xi_{2} \backslash O_{1}$ which contains $\left(x^{*}, 0, \sigma\right)$.

We take $B=B_{1} \cup O_{1}$. Therefore we can find a bounded domain $K \subset \Omega_{2}$ with smooth boundary $\partial K$ which depends on $m_{1}$ and $M$ such that $K \subset B$ (see Figure 2).

Let $L \subset K \cap\{y=0\}$ be a segment passing the point

$$
P=\left(x^{*}, 0, F_{2}\left(x^{*}, 0\right)-d_{1} \frac{1-\sin \theta}{\sin \theta}\right)
$$

such that the angle $\theta_{1}$ between the line $x=x^{*}, y=0$ and the line $L$ is less than $\frac{\theta}{2}$ (see Figure 3).

We assume that $L$ can be expressed as

$$
\begin{aligned}
x & =a_{1} t+x^{*}, \\
y & =0, \\
z & =a_{3} t+F_{2}\left(x^{*}, 0\right)-d_{1} \frac{1-\sin \theta}{\sin \theta},
\end{aligned}
$$

where $t>0$ is a parameter, $0<t<\cos \frac{\theta}{2}\left(F_{2}\left(x^{*}, 0\right)-d_{1} \frac{1-\sin \theta}{\sin \theta}\right)$ and $a_{1}^{2}+a_{3}^{2}=1$. 


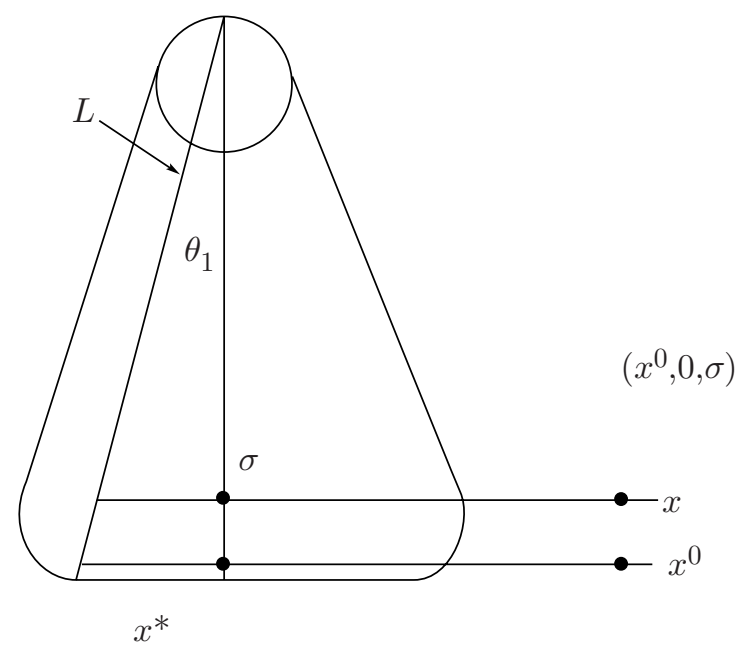

Figure 3. A line $L$ passing $P$ on the plane $y=0$

Henceforth we set

$$
R_{2}=\cos \frac{\theta}{2}\left(F_{2}\left(x^{*}, 0\right)-d_{1} \frac{1-\sin \theta}{\sin \theta}\right)
$$

Since $u_{2}$ is harmonic in $K$, by Green's formula (e.g. [1]), we have

$$
\begin{aligned}
u_{2}(x, y, z)= & \int_{\partial K}\left[G\left(x, y, z, x_{1}, y_{1}, z_{1}\right) \frac{\partial u_{2}}{\partial n}\left(x_{1}, y_{1}, z_{1}\right)\right. \\
& \left.-\frac{\partial G}{\partial n}\left(x, y, z, x_{1}, y_{1}, z_{1}\right) u_{2}\left(x_{1}, y_{1}, z_{1}\right)\right] d S, \\
& \quad \text { or } \quad(x, y, z) \in K .
\end{aligned}
$$

Here $G$ is the same as the one in (4.27).

Similarly to (4.27) in the proof of Lemma 3.1, we know that there exists a holomorphic function $v(\zeta)$ in $E_{1}=\left\{\zeta|\quad| \zeta|<b,| \arg \zeta \mid<\theta_{1}\right\}$ such that

$$
|v(\zeta)| \leq C_{10}(M), \quad \zeta \in E_{1},
$$

and

(5.6) $v(\zeta)=u_{2}\left(a_{1} \zeta+x^{*}, 0, a_{3} \zeta+F_{2}\left(x^{*}, 0\right)-d_{1} \frac{1-\sin \theta}{\sin \theta}\right), \quad$ for $\quad 0<\zeta<R_{2}$.

Here we note that $b>0$ is dependent on $\mathcal{F}$, but independent of the choice of $L$.

Here and henceforth for simplicity we can identify $E_{1}$ and the sector $\widetilde{E}_{1}$ whose vertex and angle are $P$ and $2 \theta_{1}$, respectively, because $\zeta \in E_{1}$ is transformed isometrically onto $\tilde{\zeta} \in \tilde{E}_{1}$.

We set

$$
q=\cos \frac{\theta}{2} \sin \theta
$$

From (5.1) and (5.6), noting that $O_{1} \cap L \in \mathcal{D}$ and the length of $O_{1} \cap L$ is

$$
2 d_{1} \cos \frac{\theta}{2}=q d,
$$


we have $q d<b$ and

$$
|v(\zeta)| \leq \delta, \quad \Im \zeta=0, \quad 0<\zeta<q d .
$$

In view of (5.5) and (5.7), we apply Lemma 4.4 in $\tilde{E}_{1}$ to see that

$$
\begin{aligned}
& \left|u_{2}\left(a_{1} \zeta+x^{*}, 0, a_{3} \zeta+F_{2}\left(x^{*}, 0\right)-d_{1} \frac{1-\sin \theta}{\sin \theta}\right)\right|=|v(\zeta)| \\
& \leq C_{10}(M) \delta^{C_{4}\left(\left(\frac{q d}{\zeta}\right)^{\frac{\pi}{\theta}}-\left(\frac{q d}{R_{2}}\right)^{\frac{\pi}{\theta}}\right)} \\
& \leq C_{11}(M) \delta^{C_{11} d^{\tau_{3}}}, \quad q d \leq \zeta \leq R_{2},
\end{aligned}
$$

where $\tau_{3}=\frac{\pi}{\theta}$ and $C_{4}>0$ is a constant which depends on $\tilde{E}_{1}, q d$ and $b$.

Moreover, as is seen from the proof of Lemma 4.2 in (5.5), we can find a constant $C_{4}>0$ which can be chosen uniformly in $\theta_{1}, q$ and $d$ as long as $\theta_{1}$ varies over a fixed closed interval in $\left(0, \frac{\theta}{2}\right)$.

In (5.8), we can change directions $\left(a_{1}, 0, a_{3}\right)$ of the segment $L$ such that $\theta_{1}<\frac{\theta}{2}$ and we obtain (5.3) for a sufficiently small $\kappa>0$ and some $C_{8}>0, C_{9}>0$.

Now we will complete the proof of Lemma 3.2

We apply the conditional stability for line unique continuation by Cheng, Hon and Yamamoto [8] and, Cheng and Yamamoto [9].

Lemma 5.1. Suppose that $\Omega$ is a domain in $\mathcal{R}^{n}(n \geq 3)$ and a straight line $L$ intersects $\partial \Omega$ at two points, and $\Gamma=L \cap \Omega$ is a segment. We assume that two segments $\gamma, \Gamma_{1}$ satisfy

$$
\gamma \subset \Gamma_{1} \subset \bar{\Gamma}_{1} \subset \Gamma, \quad \bar{\Gamma}_{1} \neq \Gamma .
$$

Let $u \in C^{2}(\Omega)$ be a harmonic function. If there exists a constant $M>0$ such that

$$
\|u\|_{C(\Omega)} \leq M
$$

then

$$
\|u\|_{C\left(\bar{\Gamma}_{1}\right)} \leq C\|u\|_{C(\bar{\gamma})}^{\alpha}
$$

Here $C=C\left(M, \gamma, \gamma_{1}\right)$ is a positive constant which depends on $M, \gamma$ and $\Gamma_{1}$, and $\alpha \in(0,1)$ depends on $\Gamma_{1}$ and $\gamma$. Moreover $C>0$ and $\alpha \in(0,1)$ can be chosen uniformly as long as the length $|\gamma|>\epsilon_{0}$ and $\inf _{\zeta \in \partial \Omega, \eta \in \Gamma_{1}}|\zeta-\eta|>\epsilon_{0}$ hold true for $\epsilon_{0}$.

Therefore, applying Lemma 5.1 , we can choose constants $C_{12}>0$ and $0<\beta_{1}<1$ depending on $M$ and $\kappa$ such that

$$
\left|u_{2}\left(x^{0}, 0, \sigma\right)\right| \leq C_{12} \delta_{1}^{\beta_{1}} .
$$

Hence by (5.2) and (5.3), we have

$$
\frac{m_{2}}{2} \leq\left|u_{2}\left(x^{0}, 0, \sigma\right)\right| \leq C_{12}\left(C_{8} \delta^{C_{9} d^{\frac{\pi}{\theta}}}\right)^{\beta_{1}}=C_{13} \delta^{C_{14} d^{\frac{\pi}{d}}} .
$$

Hence by noting that we may assume that $\delta>0$ is small and $0<\delta<1$ from (5.13), there exists a constant $C_{15}>0$ which depends on $m_{0}, m_{1}, m_{2}$ and $M$ such that

$$
d^{\frac{\pi}{\theta}} \leq \frac{C_{15}}{|\ln \delta|}
$$


that is

$$
d \leq C\left[\frac{1}{\log \frac{1}{\delta}}\right]^{\tau}
$$

where a constant $C>0$ is independent of $d$. Thus the proof is complete.

\section{REFERENCES}

1. G. Alessandrini, Stable determination of a crack from boundary measurements. Proc. R. Soc. Edinburgh V.123A (1993), 497-516. MR 94h:35259

2. S. Andrieux, A.B. Abda \& M. Jaoua, Identifiabilité de frontière inaccessible par des mesures de surface. C. R. Acad. Sci. Paris Sér. I Math. V.316 (1993), 429-434. MR 94b:35292

3. N.D. Aparicio \& M.K. Pidcock, The boundary inverse problem for the Laplace equation in two dimensions. Inverse Problems V.12 (1996), 565-577. MR 97h:35231

4. E. Beretta \& S. Vessella, Stable determination of boundaries from Cauchy data. SIAM J. Math. Anal. V.30 (1998), 220-232. MR 99j:35229

5. A.L. Bukhgeim, J. Cheng \& M. Yamamoto, Stability for an inverse boundary problem of determining a part of a boundary. Inverse Problems V.15 (1999), 1021-1032. MR 2000e:35235

6. A.L. Bukhgeim, J. Cheng \& M. Yamamoto, Uniqueness and stability for an inverse problem of determining a part of boundary. Inverse Problems in Engineering Mechanics (Nagano, 1998), 327-336, Elsevier, Oxford, 1998. MR 99m:35259

7. A.L. Bukhgeim, J. Cheng \& M. Yamamoto, On a sharp estimate in a non-destructive testing: determination of unknown boundaries. Applied Electromagnetism and Mechanics. K. Miya, M. Yamamoto and Nguyen Xuan Hung eds. JSAEM (1998), 64-75.

8. J. Cheng, Y.C. Hon \& M. Yamamoto, Stability in line unique continuation of harmonic functions: general dimensions. J. Inverse and Ill-posed Problems V.6 (1998), 319-326. MR 2000b:35034

9. J. Cheng \& M. Yamamoto, Unique continuation on a line for harmonic functions. Inverse Problems V.14 (1998), 869-882. MR 99d:35029

10. A. Friedman \& M. Vogelius, Determining cracks by boundary measurements. Indiana Math. J. V.38 (1989), 527-556. MR 91b:35109

11. D. Gilbarg \& N. Trudinger, Elliptic partial differential equations of second order, 2nd ed., Berlin, New York, Springer-Verlag, 1983. MR 86e:35035

12. V. Isakov, Stability estimates for obstacles in inverse scattering. J. of Computational and Applied Math. V.42 (1992), 79-88. CMP 93:01

13. V. Isakov, New stability results for soft obstacles in inverse scattering. Inverse Problems V.9 (1993), 535-543. MR 94k:35189

14. P.G. Kaup, F. Santosa \& M. Vogelius, Method for imaging corrosion damage in thin plates from electrostatic data. Inverse Problems V.12 (1996), 279-293.

15. O.D. Kellogg, Foundations of Potential Theory. Dover Publications, Inc., New York (1953). MR 36:5369

16. E. Landis, Some problems of the qualitative theory of second order elliptic equations. Russian Math. Surveys V.18 (1963), 1-62. MR 27:435

17. M.M. Lavrentiev, Some Improperly Posed Problems of Mathematical Physics. (English translation) Springer-Verlag, Berlin (1967).

18. M. McIver, Characterization of surface-breaking cracks in metal sheets by using AC electric fields. Proc. R. Soc. London A V.421 (1989), 179-194.

19. D.H. Micheal, R.T. Waechter \& R. Collins, The measurement of surface cracks in metals by using a.c. electric fields. Proc. R. Soc. London A V.381 (1982), 139-157.

20. S. Mizohata, The Theory of Partial Differential Equations. Cambridge University Press, London (1973). MR 58:29033

21. L.E. Payne, Bounds in the Cauchy problem for Laplace's equation. Arch. Rational Mech. Anal. V.5 (1960), 35-45. MR 22:1743

22. M.H. Protter \& H.F. Weinberger, Maximum Principles in Differential Equations. Englewood Cliffes, New Jersey (1967). MR 36:2935

23. A.G. Ramm, Stability of the solution to inverse obstacle scattering problem. J. Inverse and Ill-posed Problems V.2 (1994), 269-275. MR 95f:35092 
24. L. Rondi, Uniqueness and stability for the determination of boundary defects by electrostatic measurements. Ref. S.I.S.S.A. 73/98/AF (July, 1998), SISSA ISAS Trieste, Italy. CMP 2001:05

25. L. Rondi, Optimal stability estimates for the determination of defects by electrostatic measurements. Inverse Problems V. 15 (1999), 1193-1212. MR 2000k:78026

26. R. Siegel, Boundary perturbation method for free boundary problem in convectively cooled continuous casting. Trans. ASME. Sec.C, V.108-1 (1986), 230-235.

27. A.N. Tikhonov \& V.Y. Arsenin, Solutions of Ill-posed Problems. English Translation. Winston \& Sons, Washington (1977). MR 56:13604]

Department of Mathematics, Fudan University, Shanghai 200433, China \& Department of Mathematics, Faculty of Engineering, Gunma University, Kiryu 376-8515, Japan

E-mail address: jcheng@math.sci.gunma-u.ac.jp and jcheng@fudan.edu.cn

Department of Mathematics, City University of Hong Kong, Tat Chee Avenue, Kowloon, Hong Kong

E-mail address: maychon@cityu.edu.hk

Department of Mathematical Sciences, University of Tokyo, 3-8-1 Komaba, Meguro, TOKYO 153-8914, JAPAN

E-mail address: myama@ms.u-tokyo.ac.jp 